\title{
THE GENETICS OF ESSENTIAL HYPERTENSION
}

\begin{tabular}{|l|l|l|}
\hline J.A.F. & Fraser & Roberts \\
\hline
\end{tabular}

Clinical Genetics Research Unit, Institute of Child Health, the Hospital for Sick Children, Great Ormond Street, London

This paper was an addendum to that of Dr. Miall and Mr. Oldham, who included a summary of results already published by Professor Pickering, Dr. Sowry, Dr. Hamilton and myself. The paper read was largely a commentary on lantern slides, so an abstract is given here.

The original work was chiefly concerned with two samples of measurements of arterial pressures, (1) 2,000 out-patients attending dermatological, orthopaedic clinics etc., called the "population sample". (2) 1,000 first degree relatives of hypertensive subjects, who included a large number recorded in full in a monograph by Dr. Søbye. As is always found, the frequency distributions were distinctly non-normal, showing marked positive skewness and marked positive kurtosis, making it somewhat difficult to decide about the presence or absence of bimodality.

Re-examination of the figures has shown that the frequency distributions of the two samples, including both systolic and diastolic pressures, are very closely normal when the units of measurement are transformed to a logarithmic scale. It has been pointed out, notably by Gaddum, that most biological measurements tend to be more normally distributed on a logarithmic scale than on a linear scale. Hence the present examination of the figures supports the conclusion that there is no evidence of bimodality, and strengthens the theses, first, that essential hypertension is no more than the positive end of the normal distribution of arterial pressures in the population and, second, that the genetic element in hypertension is no more than the tendency for relatives to resemble each other in pattern of arterial pressure-a tendency which is quantitatively the same at all levels from highest to lowest.

The full details of this work will be published in Clinical Science. 